\title{
Influence of initial substratum surface tension on marine micro- and macro-fouling in the Gulf of Thailand
}

\author{
Klaus Becker ${ }^{*}$, Sittiphun Siriratanachai ${ }^{2} \&$ Twee Hormchong $^{3}$ \\ 'Biolab Research Institute, Kieler Straße 51, D - 24594 Hohenwestedt, Germany \\ ${ }^{2}$ Burapha University, Faculty of Science, Bangsaen-Chonburi 20131, Thailand \\ 'Siam University, Faculty of Science, 235 Phetkasem Rd., Bangkok 10163, Thailand
}

\begin{abstract}
The density of five major groups of fouling organisms fbacteria, diatoms, chodnotlagellates, ciliates, macroorganisms) on seven artificial substrata with surface tensions between 19.0 and $64.5 \mathrm{mN} \mathrm{m}$ ' was studied in the Ciulf of Thailand. Two series of test panels of the different substrata were immersed into the sea between 3 hours and fit days (macrofauna 128 days). The results show that surface tension has a limited impact on the density of the organisms. Only bacteria settled continuously in significantly lower numbers on materials within the minimum bioddhesive range (20-2.5 $\mathrm{mN}$ m $)$ than on other substrata. Significant differences between the substrata may disappear after long exposure, as in series 2 after 16 days. For diatoms and protozod, a colonisation pattern similar to that of bacteria with a minimum of $20-25 \mathrm{miN} \mathrm{m}:$ was detected after several exposure intervals. However, it was never recorded in more than 3 exposure intervals in a row. The colonisation pattern of macroorganisms could not be attributed to substratum surface tension. An index, called "colonisation degree" is introduced to give a general impression of the density of organisms on the materials tested. The colonisation degree did not show any significant difference at any exposure interval. The present results clearly suggest that substratum surface tension is easily overshadowed by other factors in colonisation processes under natural conditions.
\end{abstract}

\section{INTRODUCTION}

Marine fouling is a widespread nuisance to marine technology. Surtaces exposed to natural seawater are colonised by a large variety of organisms. The major groups are bacteria, diatoms, protozoa, and macroorganisms. Toxic paints are the most widespread antifouling devices, although they cause serious damage to the environment and aquaculture, particularly in enclosed coastal areas (Fischer et al., 1984; Cleary \& Stebbing, 1987). Among others, substratum surface tension (SI-unit: $\gamma=\mathrm{mN} \mathrm{m}^{-1}$ ) has attracted some interest as one potential non-toxic measure to prevent fouling. Substratum surface tension describes the "energetic state" of a surface. It is a major surface property that influences attachment strength of organisms. However, there is considerable uncertainty on the relevance of surface tension on the colonisation density of all fouling

- Addressee for all correspondence.

(1) Biologische Anstalt Helgoland, Hamburg 
groups under natural conditions. Several studies reported the existence of a minimum bioadhesive range on surfaces between 20 and $25 \mathrm{mN} \mathrm{m}^{-1}$ (Baier, 1973; Dexter, 1979; Goupil et al., 1980; Characklis \& Cooksey, 1983; Meyer et al., 1988; Rittle et al., 1990). Dexter (1979) proposed a thermodynamic model which explains that adhesion in seawater is minimal between 20 and $25 \mathrm{mN} \mathrm{m}$ '. Other studies found that attachment strength and colonisation density decreased with increasing surface tension (Eiben, 1976; Fletcher \& Loeb, 1979; Absolom et al., 1983; Mihm et al., 1981; van Pelt et al., 1985; Rittschof \& Costlow, 1989; Burchard et al., 1990; Roberts et al., 1991) or increased with surface tension (Absolom et al., 1983; Becka \& Loeb, 1984; Fletcher \& Baier, 1984; Crisp et al., 1985; Udhayakumar \& Karande, 1986; Rittschof \& Costlow, 1989; Roberts et al., 1991; Becker, 1993). Some studies indicate that surface tension responses differ from species to species. In some instances, densities of colonisers may even be independent of surface tension (Absolom et al., 1983; Rittschof \& Costlow, 1989; Roberts et al., 1991). Absolom et al. (1983) suggested that different substratum responses depend on whether surface tension of the organism (or its adhesives) is higher, lower, or equal to the surfare tension of the medium.

Most previous studies on the effects of surface tension on density of fouling organisms are laboratory studies, short-term field studies (maximum 2 weeks), or focussed only on selected species. Woodin (1986) reported that macrofauna larvae may show opposite reactions to one environmental factor under laboratory and field conditions Once a material is exposed to the sea, its surface tension is modified by adsorption of macromolecules and early colonisers (Baier et al., 1968; Loeb \& Neihof, 1975; Coupil et al., 1980). Although late colonisers may not meet the initial substratum surface tension, it may exert an indirect effect on these colonisers. Composition and structure of adsorbed molecular films may differ between the substrata. Mihm et al. (1981) demonstrated that the presence of a microbial film on a surface altered substratum preferences of Bugula neritina (Bryozod). A 64-day study by Becker \& Wahl (1991) in the Baltic Sed considered all major fouling groups under natural conditions. This study suggests that microfouling (bacterid, diatoms, protozoa) may be affected by surface tension accorcting to Dexter's model (1979) during early exposure intervals but that effects may disappear after long-term exposure. Results on macroorganisms yielded no clear results, partly due to slow and sparse settlement. Only one material (Parafilm) within the minimum range (20-25 $\mathrm{mN} \mathrm{m}$ ') was investigated. However, that material was very attractive to paraffin degrading bacteria, which may have overshadowed surface tension effects. Therefore, more and inert materials within the $20-25 \mathrm{mN} \mathrm{m}^{\prime}$ range should be selected. Secondly, studies should be conducted in an area with strong fouling pressure by all fouling groups. An efficient antifouling device in marine technology should be effective under heavy fouling pressure over a long period.

The aims of the present study were:

- Does initial substratum surface tension have any implication on the density of the major fouling groups under natural conditions?

- How long does initial substratum tension have an effect on the density of fouling organisms?

- Are all fouling groups affected by initial surface tension in the same or in different ways? 


\section{MATERIAL AND METHODS}

\section{Selection of substrate and determination of surface tension}

Seven artificial materials were used in the present study. Four types of fluorpolymers were provided by Hoechst Co. (Frankfurt, Germany), namely PTFE (polytetrafluorethylene), PFA (a copolymer made from PTFE and perfluorcompounds), FEP (fluorethylenepropylene), and ETFE (ethylenetetrafluorethylene). The other three materials were $\mathrm{HC}$ (an acetalpolymer, Hoechst Co.), PC (polycarbonate, Richter Co., Kiel, Germany) and glass. All substrates were inert, transparent or white, and smooth. Substratum surface tension was determined by contact angle measurements with bidistilled water and analytical grade glycerin as described earlier (Becker \& Wahl, 1991; Becker, 1993). Calculation of surface tension or surface free energy ${ }^{*}\left(\gamma_{s}\right)$ from contact angle data was made through the equation-of-state approach (Neumann et al., 1974, 1980). Prior to contact angle measurements and immersion into the sea, test panels were cleaned thoroughly by a method based on that of Busscher (1985).

\section{Experimental design of colonisation experiments}

Two panel sizes were exposed to monitor the colonisation by fouling organisms. Microfouling (bacteria, diatoms, protozod) were enumerated on $1-\mathrm{cm}^{2}$ panels which were exposed between 3 hours and 64 days in the sea. The density of each microlouling group was counted on 3 replicate panels after each exposure interval. All replicate samples for one exposure interval were stuck on one white polyamide-plate $(15 \mathrm{~cm} \times 18 \mathrm{~cm})$. They were randomly distributed at the same level with no gaps between. $25-\mathrm{cm}^{2}$ panels were used to determine the density of macroorganisms. They were exposed from 3 hours to 128 days. One panel of each material was vertically arranged and fixed between two bars. Samples were immersed into the sea close to Laem Than Beach (Chonburi Province, Gulf of Thailand) at a depth of $1.5 \mathrm{~m}$ at low tide $(4.5 \mathrm{~m}$ at high tide). They were attached to a rope between concrete poles and hung perpendicular in the water. Two experimental series were started at the same location (an illuminated position) on 08.09.91 and 30.01.92. Samples were collected at random dfter 3 and 6 hours, and after $1,2,5,8,32,64$, and 128 days. Two replicates per material were collected after each interval. Early substratum modification (3-96 h) was recorded on test panels ( 3 replicates) which were immersed into the sea. Panels were air dried (Yamato DS-62, $30^{\circ} \mathrm{C}$ ) and surface tension was determined as described above.

\footnotetext{
- There are different concepts referring to surface tension. The present study uses the concept of surface free energy at interfaces $\left(\gamma_{w}, s=\right.$ solid, $v=$ vapor; see Neumann et al., 1974) like Absolom et al. (1983). Fletcher \& Pringle (1985) and van Pelt et al. (1985). Another concept which will be of some relevance in that study is the concept of critical surface tension ( $\gamma_{r}$, see Zisman, 1964). CritiCal surface tension is an empirically determined parameter that is related to the surface tree energy of a substratum. Dexter (1979), Baier (1973), Meyer et al. (1988), Goupil et al. (1980) use the concept of critical surface tension. Nevertheless, both concepts may yjeld similar results because the values of the two terms approximate if the substrate are apolar to some extent (Rabel, $1971)$.
} 


\section{Enumeration of organisms}

After collection, the samples were preserved in $4 \%$-Formalin in artificial seawater and rinsed with distilled water to remove unattached organisms. Fouling organisms were counted on 3 replicates per exposure interval. Bacteria were stained with acridine orange and counted in 20 randomly selected fields through an epifluorescence microscope at $100 \times$ magnification. Diatoms and protozoa were stained with Alcian blue and Ziehl Neelsens stain. Their density was determined through a light microscope by counts in 15 randomly selected fields at $40 \times$ magnification. The densities of the 10 most abundant diatom genera were estimated semi-quantitatively; (a) dominant: occurred in $>75 \%$ of fields counted and was the most abundant genus in $>50 \%$ of the fields counted, (b) abundant: occurred in $>75 \%$ of fields counted and was the most abundant genus in $<50 \%$, of the fields counted, (c) regularly: occurred in $25 \%<\mathrm{x}<$ $75 \%$ of the fields counted but was never the most abundant genus, (d) seldom: occurred in $<25 \%$ of the fields counted but was never the most abundant genus. Protozoa were differentiated into choanoflagellates and ciliates. Ciliata were identified to the genus level and each genus was counted individually. Macroorganisms were identified and counted through a stereomicroscope. Coverage of the test panels by macroorganisms was determined by the dot method adopted from random sampling systems (Nair et al., 1984). On a transparent plastic sheet, $5 \times 5 \mathrm{~cm}^{2}$ were marked with minute black spots at intervals of $0.25 \mathrm{~cm}$. This sheet was randomly placed on the panels. The total number of dots covering the panel and the number of dots covering colonisers were counted. This procedure was repeated 3 times.

\section{Statistical analyses}

Statistical analyses were carried out using CSS-Statistica (Statsoft Inc.) software package. Data were log-transformed for ANOVA. A Tukey-HSD-test was employed for post-hoc comparisons of mean densities of the fouling groups on the substrata. An index called "colonisation degree" was used to get an estimate of the colonisation by all groups of fouling organisms (bacteria, diatoms, protozoa, macroorganisms) in both experimental series. The "colonisation degree" was calculated as follows: The highest mean density of each fouling group found on one material was set as $100 \%$. Densities on the other materials were calculated accordingly in $\%$-values. The average of the values for the 5 fouling groups (bacteria, diatoms, choanoflagellates, ciliates, macroorganisms) yielded the "colonisation degree".

\section{RESULTS}

\section{Surface tension of materials}

PTFE (19.0 $\left.\mathrm{mN} \mathrm{m}^{-1}\right)$ possesses the lowest surface tension while glass $\left(64.5 \mathrm{mN} \mathrm{m}^{-1}\right)$ shows the highest surface tension of the substrata investigated. FEP $\left(20.5 \mathrm{mN} \mathrm{m}^{-1}\right)$ and PFA $\left(22.0 \mathrm{mN} \mathrm{m}^{-1}\right)$ are within the proposed minimum bioadhesive range $(20-25 \mathrm{mN}$ $\left.\mathrm{m}^{-1}\right)$. ETFE $\left(25.5 \mathrm{mN} \mathrm{m}^{-1}\right)$ is slightly above this range. Surface tensions of $\mathrm{HC}(30.0 \mathrm{mN}$ $\left.\mathrm{m}^{-1}\right)$ and $\mathrm{PC}\left(33.5 \mathrm{mN} \mathrm{m}^{-1}\right)$ belong to the proposed bioadhesive range (30-40 $\mathrm{mN} \mathrm{m}^{-1}$ ) 

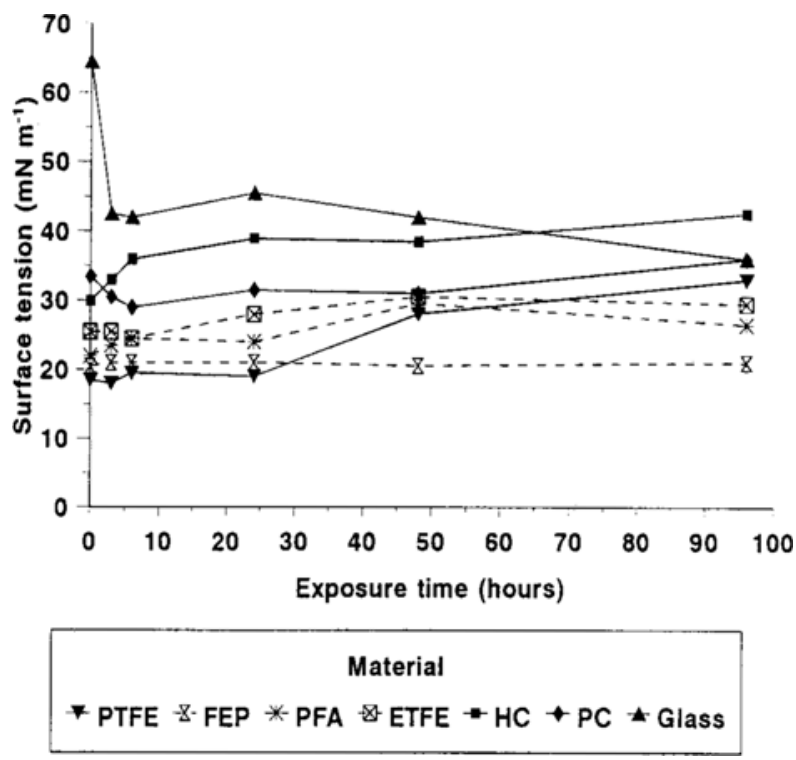

Fig. 1. Modification of initial substratum surface tension upon exposure to seawater

which otters favorable thermodynamic conditions for attachment in the sea (Baier et al., 1968; Dexter, 1979). Initial surface tension of glass declined rapidly upon immersion (Figure 1). Surface tension of PC decreased initially but later increased with exposure time. HC, PTFE, PFA, and ETFA showed increasing surface tension after exposure to seawater but PFA and ETFE remained below $30 \mathrm{mN} \mathrm{m}^{-1}$ after 96 hours. Surface tension of FEP remained fairly constant until 96 hours.

\section{Colonisation of the substrata}

None of the fouling groups investigated showed in- or decreasing densities with increasing surface tension. A colonisation pattern according to the model by Dexter (1979) could be found several times. However, a clear influence of surface tension on the density of the fouling groups could only be confirmed for bacteria (Table 1. Figure 2a). Lowest numbers were usually found on FEP $\left(20.5 \mathrm{mN} \mathrm{m}^{-1}\right)$, PFA $\left(22.0 \mathrm{mN} \mathrm{m}^{-1}\right)$ or ETFE $\left(25.5 \mathrm{mN} \mathrm{m}^{-1}\right)$ except in series 2 after 6 hours and 64 days when glass was the least densely colonised material. Significant differences (Tukey-HSD-test: $p<0.05$ ) between the substrata were observed until 64 days (series 1) and 8 days (series 2), respectively. Significant differences between the substrata were always recorded between at least one material within the $20-25 \mathrm{mN} \mathrm{m}^{1}$ range (FEP, PFA, ETFE) and PTFE as well as HC or PC. These results support the model by Dexter (1979) for bacteria although significant differences may disappear after longer exposure intervals as in series 2 . The observation that glass $\left(64.5 \mathrm{mN} \mathrm{m}^{-1}\right)$ was often less densely colonised than $\mathrm{HC}\left(30.0 \mathrm{mN} \mathrm{m}^{-1}\right)$ and PC $\left(33.5 \mathrm{mN} \mathrm{m}^{-1}\right)$ complies with Dexter's model (1.97.9). It pre- 

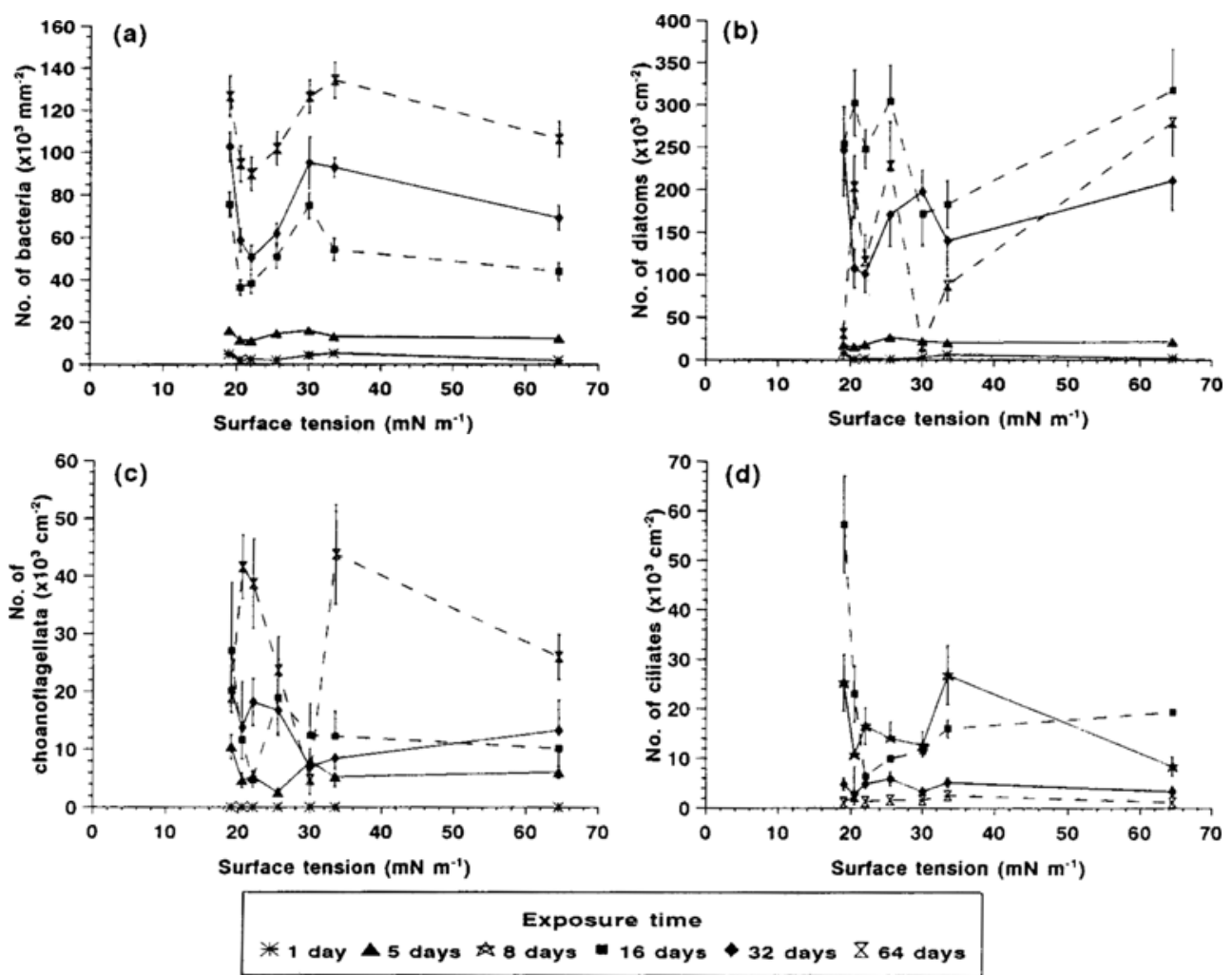

Fig. 2. Examples of colonisation patterns on the artificial materials after selected exposure intervals: (a) bacteria (series 1), (b) diatoms (series 2), (c) chodnoflagellates (series 1), (d) ciliates (series 1)

dicts declining densities above $40 \mathrm{mN} \mathrm{m}^{\prime}$ for thermodynamic reasons. Figure 2a displays the colonisation pattern of bacteria after selected exposure interval in series 1 .

However, bacteria remained the only group of fouling organisms which showed a "stable" colonisation pattern over a long period. The results for diatoms and protozoa suggest that surface tension may, to some extent, have an impact on the substratum colonisation by these organisms, but it is easily overshadowed by other factors. A colonisation pattern with lowest densities between 20 and $25 \mathrm{mN} \mathrm{m}^{-1}$ was found for diatoms in series 1 from 6 hours to 16 days, but significant differences (Tukey-HSD-test: $p<0.05$ ) were not continuously recorded (Table 2 , Figure $2 b$ ). In the 2 nd series, maximum and minimum densities were detected on different substrata from one exposure interval to the next. The 10 most abundant genera were Achnanthes, Amphora, Cocconeis, Diploneis, Grammatophora, Licmophora, Navicula, Nitzschia, Pleurosigma, and Synedra. However, according to the semiquantitative estimation of their abundance, none of these genera showed a regular colonisation pattern which could be clearly attributed to surface tension. Like diatoms, densities of choanoflagellates (Table 3, Figure 2 c) and ciliates (Table 4, Figure 2d) could not be linked clearly to surface tension 
Influence of initial substratum surface tension on fouling

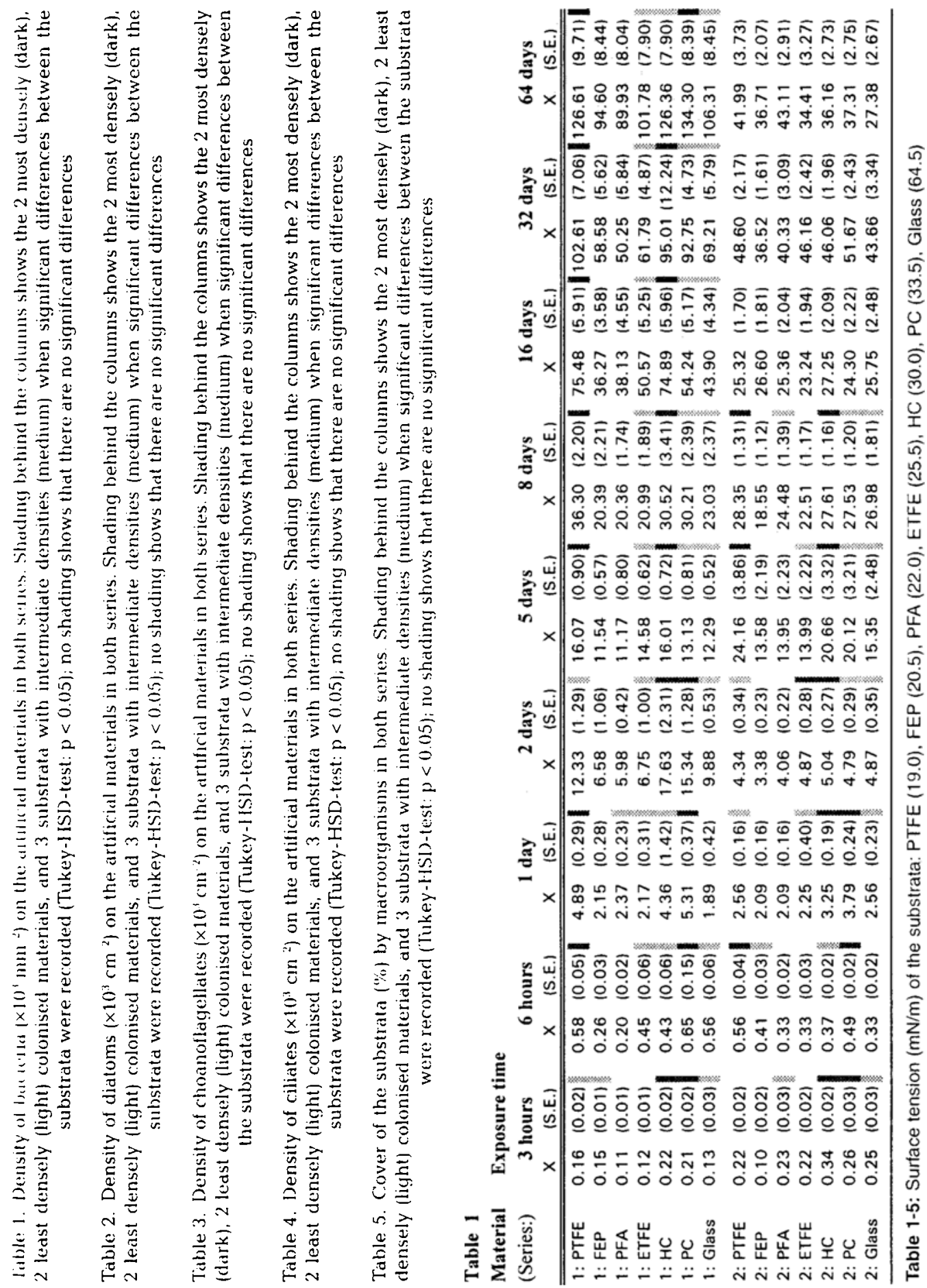




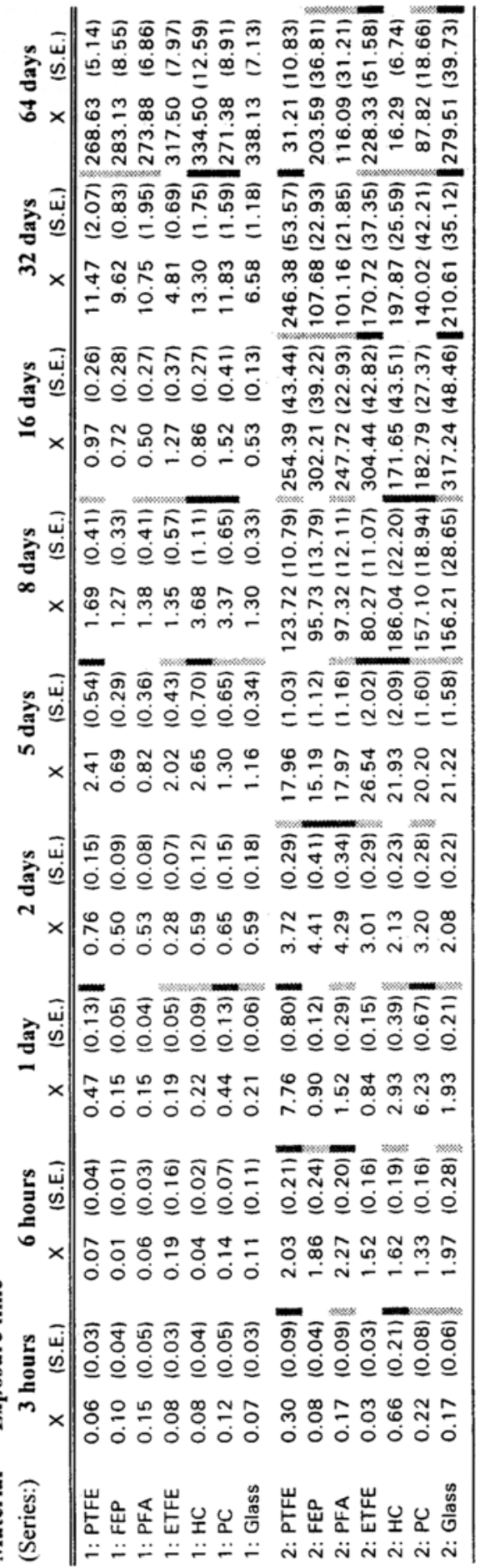

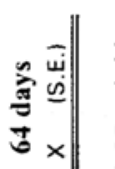

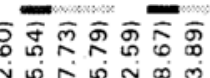
뜨늠 $\underline{\dot{\omega}}$

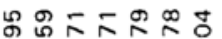
๓

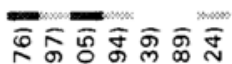
ले

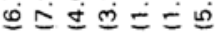
ำ능ํㅇㅇㅛ

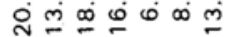

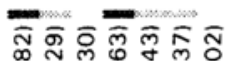
恣

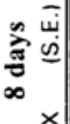
$=\ddot{m}=\underline{\dot{\omega}} \dot{\dot{U}}$

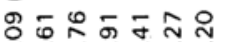

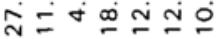
สิ

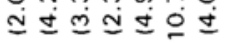

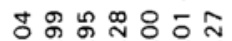

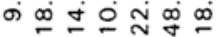

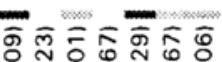
帘 $\underline{\underline{I}}=\underline{\underline{O}}=$ น

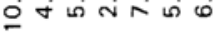

ㅎํㅇㅎㅇㅎㅎㅇㅎㅠ N ㅇํㅇํㅇ $\circ: 88: 8:$ 0000000

흫ํㅎㅎํㅎํ

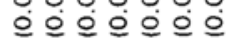
: : : : : 0000000

ํㅡㄹ

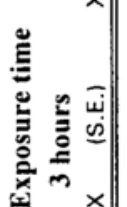

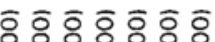

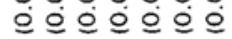
৪:৪৪:৪ 0000000 ㅎํㅇㅎํㅎํㅇㅎํㅇ 웡ㅇㅇㅇํㅇ ৪:০৪:৪৪ 0000000

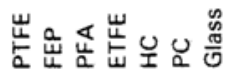

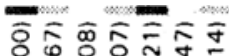

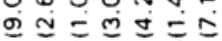

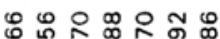

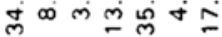

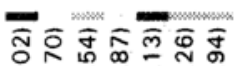

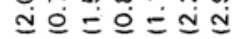
ฌํำ 총ำ \pm का

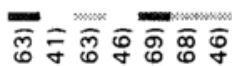
으으으워웅

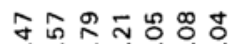
न ल

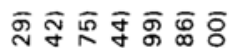
응ㅇ을

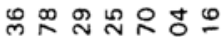
우웅응

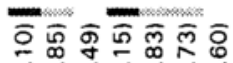
$=\underline{O} \dot{0} \dot{0} \dot{0}$

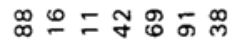
뜬ำ

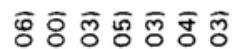
으응ํㅇํㅇ 유워ํ유. ००00000

ํํㅇํํㅇㅎํㅎํㅇ ㅇํㅇㅇํ엉 용융ㅇㅇ 0000000

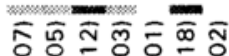

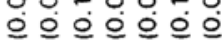
일 주요요 ०००00-0

ㅎํㅎㅎํㅎㅎㅎํำ 응ํㅇํㅇํㅇ 응ㅇㅇㅇㅇ . 000000

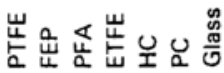
$\ddot{\sim} \ddot{\sim} \ddot{\sim} \ddot{\sim} \ddot{\sim} \ddot{N}$ 


\begin{tabular}{|c|c|c|}
\hline خِّن & 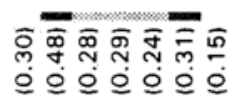 & 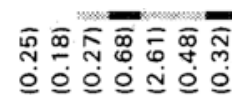 \\
\hline & 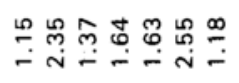 & 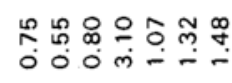 \\
\hline & 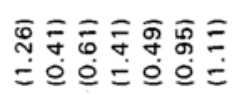 & 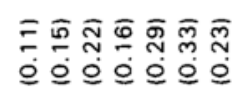 \\
\hline & 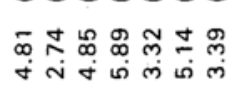 & 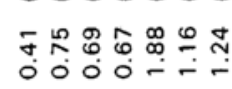 \\
\hline & 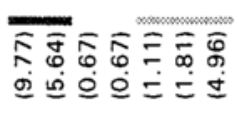 & 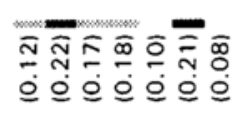 \\
\hline & 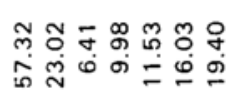 & 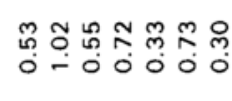 \\
\hline & 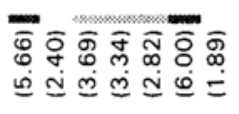 & 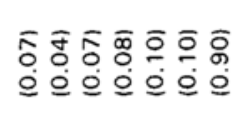 \\
\hline & 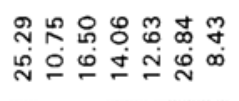 & 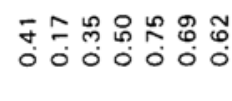 \\
\hline & 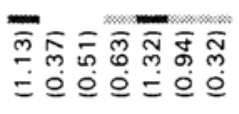 & 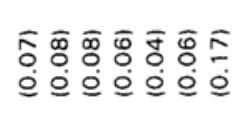 \\
\hline & 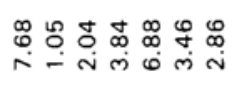 & 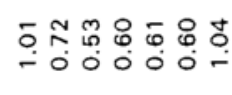 \\
\hline & 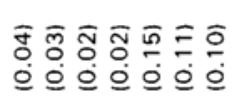 & 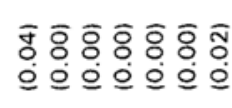 \\
\hline & 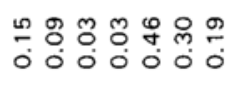 & 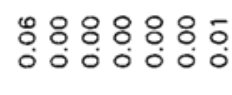 \\
\hline & 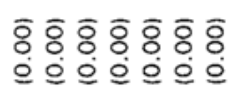 & 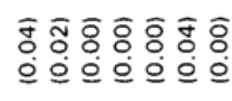 \\
\hline & 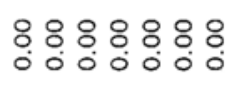 & 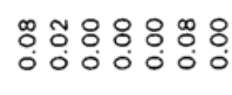 \\
\hline & 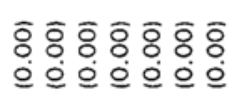 & 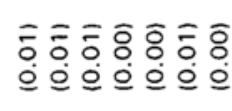 \\
\hline & 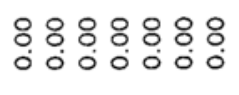 & 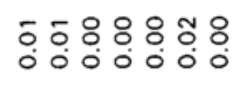 \\
\hline & 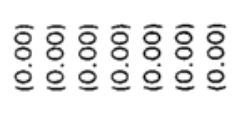 & 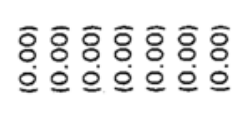 \\
\hline & $\begin{array}{l}808: 8 \% 8 \\
0000000\end{array}$ & 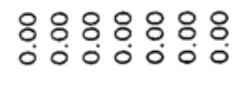 \\
\hline & 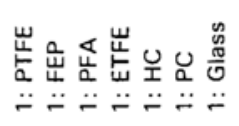 & 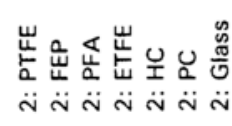 \\
\hline
\end{tabular}

\begin{tabular}{|c|c|c|}
\hline 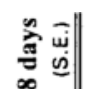 & 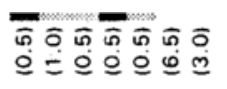 & 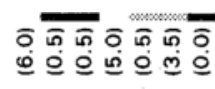 \\
\hline & ஃ & 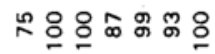 \\
\hline & 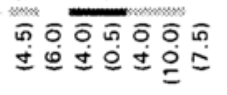 & 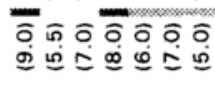 \\
\hline & 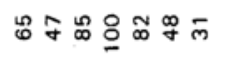 & - \\
\hline & 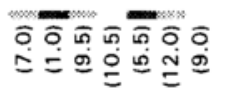 & 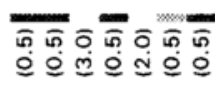 \\
\hline & స & 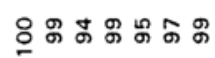 \\
\hline & 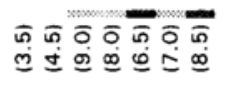 & 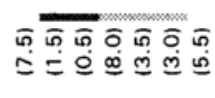 \\
\hline & స N & 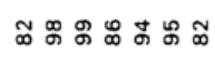 \\
\hline & 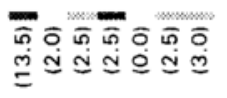 & 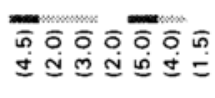 \\
\hline & $\hat{f}^{m+\sigma-N \infty}$ & 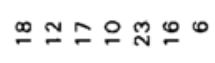 \\
\hline & 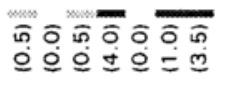 & 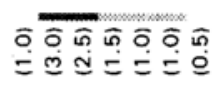 \\
\hline & nO & $N O n+\theta+N$ \\
\hline & 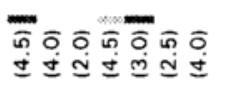 & 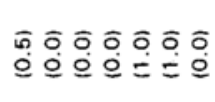 \\
\hline & $o=0=N=$ & $-000--0$ \\
\hline & 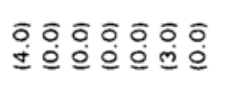 & 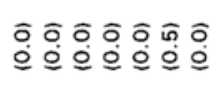 \\
\hline & 0000000 & $0000 \ldots$ \\
\hline & 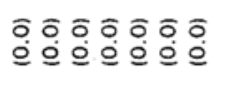 & ồ \\
\hline & 0000000 & 0000000 \\
\hline & 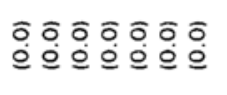 & 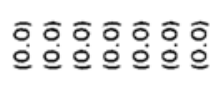 \\
\hline & 0000000 & 0000000 \\
\hline .ֶ户 & 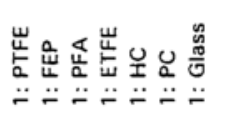 & 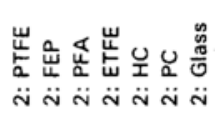 \\
\hline
\end{tabular}


although lowest numbers of both groups occurred sometimes on one material within the $20-25 \mathrm{mN} \mathrm{m}^{-1}$ range. The pattern with significant differences between the substrata did not occur over more than 2 exposure intervals except ciliates from 5 to 16 days in series 1 . The following genera could be identified; Vorticella, Zoothamnium, Epistylis, Corthunia, Pyxicola, Folliculina, Vaginicola (Peritricha), Ephelola, and Acineta (Suctoria). None of these genera showed regularly preferences towards particular substrata that would have suggested there was a significant impact of surface tension on their densities.

Among macroorganisms, 7 genera of algae (Erythrocladia sp., Ulvella sp., Chaetomorpha sp., Enteromorpha sp., Melobesia sp., Ceramium sp., Polysiphonia sp.) and 34 macrofauna species were detected. The most abundant macrofauna species were Laomedea sp. (Hydrozoa: Campanulariidae), Pomatoleios kraussii (Polychaeta: Serpulidae), Polydora normalis (Polychaeta: Spionidae), Balanus variegatus (Cirripedia: Baldnidae), and Corophium sp. (Amphipoda: Corophiidae). These species were present after most of the exposure intervals and occurred in high numbers. Except for the colonial hydrozod which covered up to $48 \%$ of the panels, barnacles were the most abundant species. B. variegatus reached densities of 205 specimen per $10 \mathrm{~cm}^{2}$ (on $\mathrm{HC}$ : Series 1. 32 days), P. kraussii $68 / 10 \mathrm{~cm}^{2}$ (ETFE: Series 1, 64 days), Corophium sp. $82 / 10 \mathrm{~cm}^{2}$ (ETFE: Series 2, 16 days), and Polydora normalis $83 / 10 \mathrm{~cm}^{2}$ (PC: Series 2, $32 \mathrm{~d}$ ). Other common species were actinians (Gen. sp.), Sabellaria c.f. spinulosa (Polychata: Sabellaridae), Perna viridis (Mollusca: Mytilidae), Crassostrea c.f. commercialis (Mollusca: Ostreidae). Their densities remained below 5 individuals per $10 \mathrm{~cm}^{2}$. The density of encrusting species (Porifera, Bryozod, Ascidiaced) remained low until 32 days in both series (cover: $\leq 10 \%)$. However, they occupied up to $100 \%$ of the available space on some panels after 64 and 128 days. Coverage of test panels by macroorganisms (Table 5, Figure 3) and colonisation patterns of individual species could not be. attributed to surface tension. Highest and lowest cover was recorded on d different substratum after almost each interval.

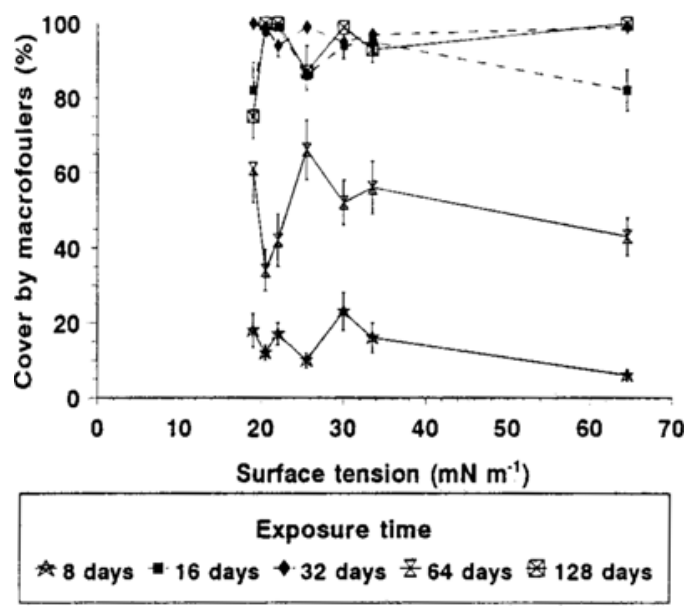

Fig. 3. Colonisation pattern of macroorganisms in series 2 


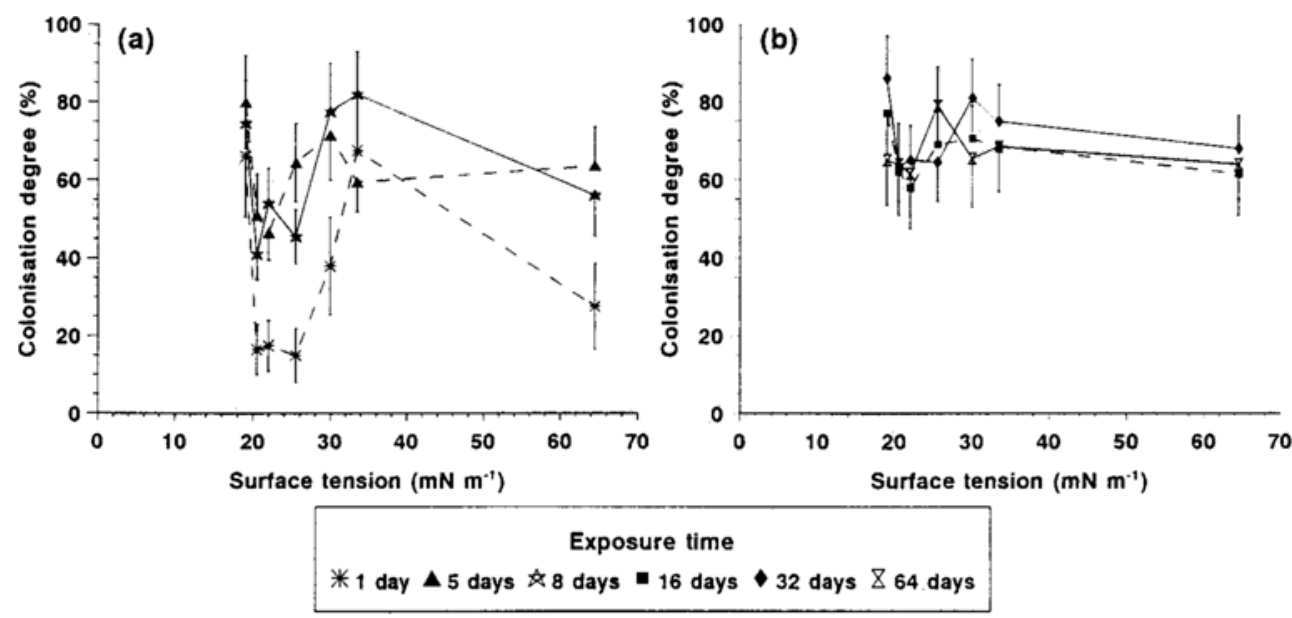

Fig. 4. Colonisation degree on the artificial materials (Summary of both series): (a) Exposure time 1, 5, 8 days; (b) Exposure time 16, 32, 64 days

The colonisation degree (Figure $4 \mathrm{a}-\mathrm{b}$ ) also reveals that surface tension is obviously not important as a fouling control device. At early exposure intervals (until 16 days), the colonisation degree remained on substrata between 20 and $25 \mathrm{~m} \wedge \mathrm{m}$ ' much lower than on the other materials. However, during initial periods of exposure the colonisation deyree is largely determined by bacteria, which fit Dexter's model at best. At early stages, other groups, namely protozod and macroorganisms, are either still lacking or their densities do not differ very much between the materials due to low densities on the test panels. Significant differences (Tukey-HSD-test: $p<0.05$ ) in the colonisation degree between the materials were only found after 8 days of exposure.

\section{DISCUSSION}

Although several studies reported substratum surface tension as an important factor in substratum colonisation (e.g., Fletcher \& Loeb, 1979, Dexter, 1979; Absolom et al., 1983; Eiben, 1976; Hsieh \& Timm, 1988; Mihm et al., 1981; Rittschof \& Costlow, 1989; Roberts et al., 1991; Lindner, 1992), the present study suggests that surface tension has a very limited impact on the density of fouling organisms under natural conditions. None of the surface tensions tested offered unfavourable conditions for attachment, strong enough to minimize surface colonisation by high detachment and low attachment rates of fouling organisms. Strong attachment to a surface enables organisms to resist strong water action and predation (Gubbay, 1983; Witman \& Suchanek, 1984; Denny, 1988). In the sea, there are various substrata with different surface properties to which organisms must adhere. Marine species obviously evolved mechanisms to prevent dislodgement from various types of natural and, according to the present study, from artificial substrata as well. 
Only bacterial colonisation patterns support the thermodynamic model by Dexter (1979) to some extent. Dexter (1979) showed the existence of a minimum bioadhesive range for bacteria on surfaces between 20 and $25 \mathrm{mN} \mathrm{m}^{-1}$. However, differences between the materials may be levelled off by longer exposure intervals. None of the other major groups of fouling organisms showed continuously a similar pattern over a long period. No evidence was found that there is a linear relationship between surface tension and density of organisms as predicted by Absolom et al. (1983) according to laboratory studies.

Marshall (1973) described bacteria as "living colloidal particles". That means bacterial adhesion is more governed by physical surface parameters of the cell envelope and substratum surface than adhesion by other (larger) organisms. Strong adhesion between surfaces occurs if adhesive and substratum have similar wettability ( $\mathrm{Wu}$, 1973). In the sea there are many strains of bacteria with different surface tensions (Busscher et al., 1984; Fattom \& Shilo, 1984; Fletcher \& Pringle, 1985). Therefore, a wide range of substratum surface tensions may be colonised by different strains of bacteria. Further, bacteria possess a variety of mechanisms to respond to different surface conditions (Rutter, 1980; Pringle \& Fletcher, 1983; Marshall, 1986; Van Loosdrecht et al., 1989). Paul \& Jeffrey (1985) and Van Loosdrecht et al. (1987) showed that one bacterial strain may switch from hydrophobic to hydrophilic attachment mechanisms depending on whether they settle on unpolar (low surface tension, $\gamma_{w}$ ) or polar (high surface tension, $\gamma_{1}$ ) surfaces. Extracellular polymers have been widely described as bacterial adhesives. These polymers are mostly composed of acidic polysaccharides (Fletcher \& Floodgate, 1973; Sutherland, 1980; Shea et al., 1991) which adhere more strongly to polar surfaces. They also often contain proteins or lipids (Fletcher \& Marshall, 1982; Parker \& Munn, 1984; Neu \& Poralla, 1988; Abu et al., 1991) which may favor attachment on hydrophobic surfaces. Some strains produce hydrophobic polysaccharides (Christensen et al., 1985; Veu \& Poralla, 1988). The variety of attachment mechanisms enable one strain to attach to different kinds of surfaces. Becker (1996) demonstrated that bacteria attached at early exposure intervals more strongly to $\mathrm{PC}\left(33.5 \mathrm{mN} \mathrm{m}^{-1}\right)$ and glass (64.5 $\mathrm{mN} \mathrm{m}$ ') than to materials within the $20-25 \mathrm{~m} .1 \mathrm{~m}$ ' range. However, bacteria achieve similar attachment strength on each material with time. Thus, bacteria adapt themselves to different surfaces and can overcome unfavorable thermodynamic surface conditions. Therefore, bacterial colonisation fits Dexter's model (1979) very well after short exposure intervals but differences may disappear. The fraction of very firmly adhering bacteria may increase with time. Duddridge et al. (1982) showed that a certain fraction of Pseudomonas fluorescens resisted shear forces of $120 \mathrm{~N} \mathrm{~m}^{-2}$ on Perspexplates although most of the cells were removed at $11 \mathrm{~N} \mathrm{~m}^{2}$.

Diatoms are also able to improve attachment strength with time on a wide range of materials (Woods \& Fletcher, 1991; Becker, 1996). Diatom glues are predominantly composed of polysaccharides (Chamberlain, 1976; Daniel et al., 1980; Cooksey \& Cooksey, 1986; Hoagland et al., 1993), but it has been shown that proteinous material is involved in attachment processes as well (Webster et al., 1985). Like bacteria, diatoms are able to sense different surface conditions (Wigglesworth-Cooksey \& Cooksey, 1992). Little is known about the composition of adhesives of protozoa, but a complex ultrastructure of stalks of sessile peritrichan ciliates and suctorians (Brown et al., 1984; Vogelbein \& Thune, 1988) indicates the existence of different components in their 
adhesives. Sulfur containing protein-polysaccharide-complexes have been detected in the freshwater suctoria Toxophyra infusionum (Hascall \& Rudzinska, 1970; Hascall, 1973). Therefore, protozoa may also overcome unfavorable thermodynamic surface. conditions by specific attachment mechanisms.

Several laboratory and short-term field studies detected considerable differences in attachment strength of macroorganisms on different substrata, and that they prefer to settle on surfaces which provide good adhesion (e.g. Eiben, 1976; Yule \& Crisp, 1983; Becka \& Loeb, 1984; Brewer, 1984; Fletcher \& Baier, 1984; Yule \& Walker, 1984, 1985; Crisp et al., 1985; Udhayakumar \& Karande, 1986; Rittschof \& Costlow, 1989; Roberts et al., 1991; Becker, 1993). However, Maki et al. (1992) found no effect of surface tension (of bacterial films) on barnacle settlement. Becker (1993) reported that the density of one barnacle and one serpulid polychaete species was not affected by substratum surface tension despite different attachment strength. In the present study, attachment strength on each material was strong enough to support dense fouling populations. A variety of other environmental factors (e.g., availability of space, nutrient supply, conspecific attraction) were obviously much more important for substratum colonisation than surface tension.

In general, highly wettable surfaces (e.g., glass) become less wettable quickly upon exposure to natural waters, and less wettable surfaces (e.g., Teflon) become more wettable to some extent. Thus, the surface tensions on various surfaces should converge due to early adsorption and colonisation processes (Baier, 1973; Coupil et al., 1980). This study indicates that initial surface tension of some materials, particularly those within the minimum bioadhesive range, remains within that range over several days. Slow alteration of surface tension of FEP $(20.5 \mathrm{mN} \mathrm{m})$ and PFA $(22 \mathrm{mN} \mathrm{m})$ can be explained by initially less compact or weaker adhering biofilms on material with the minimum bioadhesive range than on other substrate (Baier et al., 1968; Baier, 1973) or that molecular and bacterial films on the surfaces reflected properties of the underlying surface (Roberts et al., 1991). Organisms may meet with initial substratum surface tension over a few days of exposure on some materials. However, except for bacteria to some extent, neither direct effects at early intervals nor indirect effects at later exposure intervals on colonisation could be recorded.

Another result of the present study is, that lower bacterial colonisation on substrata between 20 and $25 \mathrm{mN} \mathrm{m}$ ' did not result in lower densities of late colonisers, namely protozoa and macrofauna. Mixed microbial films like those in the present study exert frequently positive effects on subsequent colonisers (e.g.. Kirchman et al., 1982; Maki et al., 1989, 1990; Cooksey \& Wigglesworth-Cooksey, 1995). If surface tension affects the colonisation via the composition of the microbial film, the effect is either very small or else bacteria and diatom densities and microbial film composition did not differ enough between the materials.

Substratum surface tension is only one property among a variety of others which influence surface colonisation under natural conditions. The present study showed that its effect on the density of fouling organisms is small. Bacteria are the sole group which were affected to some extent. A recent paper by Clarkson \& Evans (1995) also suggests that low surface tension hardly prevents heavy fouling. Griffith (1985) reported some success by applying a non-adhesive coating on a ship hull. Nevertheless, that ship had to be cleaned every $f$ months. Therefore, surface tension is of minor importance in foul- 
ing control unless new aspects are introduced. For example, Lindner (1992) reported that extremely low surface tension $\left(12 \mathrm{mN} \mathrm{m}^{-1}\right)$ offers very weak adhesion to barnacles leading to low densities in field experiments. However, such surfaces are difficult to prepare and the results have to be confirmed in future field studies.

Acknowledgements. Thanks are due to the staff of Bangsaen Institute of Marine Science (BIMS), Bangsaen-Chonburi 20131 (Thailand) for their support during this research program and to Dr. M. Wahl and Prof. S. Lorenzen (University of Kiel) for their advice and helpful discussion of this paper. Financial support was granted by the G. Daimler \& K. Benz Foundation (Germany).

\section{LITERATURE CITED}

Absolom, D. R., Lamberti, F. V., Policova, Z., Zingg, W., van Oss, C. J. \& Neumann, A. W., 1983. Surface thermodynamics of hacterial adhesion. - Appl. environ. Microbiol. 46, 90-97.

Abu, G. O., Weiner, R. M., Rice, J. \& Colwell, R. R., 1991. Properties of an extracellular adhesive polymer from the marine bacterium Schewanella colwelliana. - Biofouling 3, $6.9-84$.

Baier, R. E.. 1973. Influence of the initial surtace condition of materials on hinadhesion. In: Proceedings 3 rd International Congress on Marine Corrosion and Fouling. Ed. by R. F. Acker, B. F. Brown, J. R. DePalma \& W. P. Iverson. Vational Buredu of Standards, Gaithersburg. 633-6;39

Baier. R. E., Shafrin, E. G. \& Zisman, W. A., 1968. Adhesion: Mechanisms that assist or impede it. Science, V.Y. 162, 1360-1368.

Becka, A \& Loeb, G. 1984. Ease of removal of barnacles from various polymeric materials. Biotech. Bioeng. 26. 1245-1251.

Berker, K., 2993. Attachment strength and colonization patterr: of two marolou!ng spectes on substrata with difterent surface tension (in-situ studies). - Mar. Biol. 177. 301 -309

Becker, K., 1996. EPS-production and attachment strength of bacteria and diatoms on substrata with different surface tensions. - Microb. Ecol. 32, 23-33.

Becker, K. \& Wathl, M., 1991. Influence of substratum surface tension on biofouling of artificial substrata in Kiel Bay (Western Baltic): In-situ studies. - Biofouling 4, 275-291.

Brewer, R. H., 1984. The influence of the orientation. roughness, and wettability of solid surfaces on the behavior and attachment of planulae of Cyanea (Cndaria: Sryphozoa). - Biol. Bull. mar. biol. Lab., Woods Hole 166, 11-21.

Brown, I., Blunn, G. \& Jones, E. B. Ci., 1984. Alluchment of marune fouling Protozod. In: Proceedings 6 th International Congress of Marine Corrosion and Fouling Athens, 113-127.

Burchard, R. P. Rittschof, D. \& Bonaventura. J., 1990. Adhesion and motility of gliding bacteria on substrata with different surface free energies. - Appl. environ. Microbiol. 56, 2529-25.34

Busscher, H. J., 1985 Surface free energies and the adhesion of oral bacterici. Ph.D. Thesis, Riksuniversitet te: Groningen, $144 \mathrm{pp}$.

Busscher, H. J., Weekamp, A. H., van der Mei, H. C., van Pelt, A. W. J., de Jonge, H. P. \& Arends, J., 1984. Measurements of the surface free energy of bacterial cell surfaces and relevance for adhesion. - Appl. environ. Microbiol. 48,980-983.

Chamberlain. A. H. L., 1976. Algal settlement and secretion of acthesive materials. In: Proceedings 3rd International Biodegradation Symposium. Ed. by J. M. Sharpley \& A. M. Kaplan. Appl. Sci., London. $417-432$.

Characklıs, W. G. \& Cooksey, K. E., 1983. Biofilms and microblal fouling. - Adv. appl. Microbiol. $29.93 \cdot 138$

Christensen, B. E., Kjosbakken, J. \& Smidsrod, O., 1985. Partial chemical and physical characterization of two extracellular polysaccharides produced by marine periphytic Pseudomonas sp. strain NCMB 2021. - Appl. environ. Microbiol. 50, 837-845.

Clarkson, V. \& Evans, L. V., 1995. Raft trial experiments to investigate the antifouling potential of silicone elastomer polymers with added biocide. - Biofouling 9. 129-143.

Cleary. J. J. \& Stebbing, A. R. D., 1987. Organotin in the surface microlayer and subsurface water of southwest England. - Mar. Pollut. Bull. 48, 238-246

Cooksey, K. E. \& Cooksey, B., 1986. Adhesion of fouling diatoms to surfaces: some biochemistry. In: Algal biofouling. Ed. by L. V. Evans \& K. D. Hoagland. Elsevier, Amsterdam, 41-53. 
Cooksey, K. E. \& Wigglesworth-Cooksey, B., 1995. Adhesion of bacteria and diatoms to surfaces in the sed: d review. - Aquat. microb. Ecol. 9,87-96

Crisp, D. J., Walker, G., Young, G. A. \& Yule, A. B., 1985. Adhesion and substrate choice in mussels and barnacles. - J. Coll. Interf. Sci. 104, 40-50.

Daniel, G. F., Chamberlain, A. H. L. \& Jones, E. B. G., 1980. Ultrastructural observations on the marine fouling algae: Amphora. - Helgoländer Meeresunters. 34, 123-149.

Benny, M. W., 1988. Biology and the mechanisms of the wave swept environment. Princeton Univ. Press, New Jersey, 329 pp.

Dexter, S. C., 1979. Influence of substratum critical surface tension on bacterial adhesion - in-situ studies. - J. Coll. Interf. Sci. 70. 346-354.

Duddridge, J. E., Kent, C. A. \& Laws, J. F., 1982. Effect of surface shear stress on the attachment of Pseudomonas iluorescens to stainless steel under defined flow conditions. - Biotech. Bioeng. $24,153-164$.

Eiben, R., 1976. Der Einflul3 der Benetzungsspannung und Ionen auf die Substratbesiedlung und das Einsetzen der Metamorphose bei Bryozoenlarven (Bowerbankia gracilis). - Mar. Biol. 37 , $249-254$.

Fattom, A. \& Shilo, M., 1984. Hydrophobicity as an adhesion mechanism of benthic cyanobacteria. - Appl environ. Microbiol. 47, 135-143.

Fischer, E. C.. Castelli, V. J., Rogers, S. D. \& Beile, H. R., 1984. Technology for control of marine fouling - a review. In: Marine corrosion and biodeterioration - An interdisciplinary study. Ed. by J. D. Costlow \& R. C. Tipper. Spon, London, 261-294.

Fletcher, $M$. \& Floodgate, G. D., 1973. An electron-microscopic demonstration of an acidic polysaccharide involved in the adhesion of a marine bacterium to solid surfaces. - J. gen. Microbiol. 74 . 325-334.

Fletcher, M. \& Loeb, G. I., 1979. Influence of substratum characteristics on the attachment of a marine Pseudomonad to solid surfaces. - Appl. environ. Microbiol. 37, 67-72.

Fietcher, 3. \& Marshall. K. C., 1982. Bubble contact angle method for evaitidting sunstratum inierlacial characteristics and its relevance to hacterial attachment. - Appl. environ. Microbiol. 44. $184-192$.

Fletcher, R. L. \& Baier, R. E., 1984. Influence of surface energy on the development of the green alga Enteromorpha. - Mar. Biol. Lett. 5, 251-254

Fletcher, $\mathrm{M}$. \& Pringle, J.H., 1985. The effect of surface tree energy and medium surface tension on bacterial attachment to solid surfaces. - J. Coll. Interf. Sci. 104, 5-13

Goupil, D. W. DePalma, V. A. \& Baier, R. E., 1980. Physical/Chemical characteristics of the macromolecular conditioning film in biological fouling. In: Proceedings 5 th Congress on Marine Corrosion and Fouling. Ed. by E. C. Harderlie \& R. C Tipper. Madrid, 401-410.

Griffith, J. R., 1985. The fouling release concept: a viable alternative to toxic antifouling coatings? Trans. Inst. mar. Engrs 97, (cont. 2, paper 38), 235-235.

Gubbay, S., 1983. Compressive and adhesive strength of a variety of British barnacles. - J. mar. biol. Ass. L.K. 63, 541-555.

Hascall, G. K., 1973. The stalk of the suctorian Tokophyra iniusionum: Histochemistry, biochemistry, and physiology - J Protozool. 20, 701-704.

Hascall, G. K. \& Rudzinska, M. A., 1970. Metamorphosis in Tokophyra iniusionum; and electronmicroscope study - J. Protozool. 17, 311-323.

Hodgland, K. D.. Rosowski, J. D., Gretz, M. R. \& Roemer, S. C., 1993. Diatom extracellular polymeric substances: Function, fine structure, chemistry, and physiology. - J. Phycol. 29, 537-566.

Hsieh, Y.-L. \& Timm, D. A., 1988. Relationship of substratum wettability measurements and initial Staphylococcus aureus adhesion to films and fabrics - J Coll. Interf. Sci. 123, 275-286

Kirchman. D., Graham, S., Reish, D. \& Mitchell, R., 1982. Bacteria induce settlement and metamorphosis of Janua (Dexiospira) brasiliensis Grube (Polychaeta: Spirobided). - J. exp. mar. Biol. Ecol. 56, 153-163.

Lindner, E., 1992. A low surface energy approach in the control of marine biofouling. - Biofouling $6,193-205$.

Loeb, G. I. \& Neihof. R. A., 1975. Marine conditioning films. - Adv. Chem. Ser. 145, 319-335.

Loosdrecht van, M. C. M. Lyklema, J., Norde, W. \& Zehnder, A. J. B., 1989. Bacterial adhesion: A physicochemical approach. - Microb. Ecol. 17, 1-15.

Loosdrecht van, M. C. M., Lyklema, J., Norde, W., Schrad, G. \& Zehnder, A., 1987. Electrophoretic 
mobility and hydrophobicity as a measure to predict the initial steps of bacterial adhesion. Appl. environ. Microbiol. 53,1898-1901.

Maki, J. S., Rittschof, D., Schmidt, A. R., Snyder, A. G. \& Mitchell, R., 1989. Factors controlling attachment of bryozoan larvae: A comparision of bacterial films and unfilmed surfaces. - Biol. Bull. mar. biol. Lab. Woods Hole 177, 295-302.

Maki, J. D., Rittschof, D., Samuelsson, M.-O., Szewyk, U., Yule, A. B., Kjelleberg, S., Costlow, J. D., Mitchell, R., 1990. Elfect of marine bacteria and their exopolymers on the attachment of barnacle cyprid larvae. - Bull. mar. Sci. 46,499-511

Maki, J. S., Rittschof. D. \& Mitchell, R., 1992. Inhibition of barnacle attachment to bacterial films: An investigation of physical properties. - Microb. Ecol. 23, 97-106.

Marshall, K. C., 1973. Mechanism of adhesion of marine bacteria to surfaces. In: Proceedings 3rd International Congress on Marine Corrosion and Fouling. Ed. by R. F. Acker, B. F. Brown, J. R. DePalma \& W. P. Iverson. National Bureau of Standards, Gaithersburg, 625-b34.

Marshall, K. C., 1986. Adsorption and ddhesion processes in microbial growth at interfaces. - Adv. Coll. Interf. Sci. 25, 59-86

Meyer, A. E., Baier, R. E. \& King, R. W., 1988. Initial fouling of nontoxic coatings in fresh, brackish, and sea water. - Can. J. chem. Engng. 66, 55- $i 2$.

Mihm, J. W., Banta, W. C. \& Loeb, G., 1981. Effects of adsorbed organic and prinary fouling films on bryozoan settlement. - J. exp. mar. Biol. Ecol. 54, 167-179.

Vidir, N. B., I harmaraj, K., Abdul Azis, P. K., Arunachalam, M. \& Krishna Kumar, K., 1984. Ecology of biofouling on Crassostrea madrasensis (Preston) (Mollusca: Bivalvia) in a tropical backwater. - Proc indian Acad. Sci. (Animal Science) 93, 419-430

Veu, T. R. \& Poralla, K., 1988. An amphiphilic polysaccharide from an adhesive Rhodococcus strain. - IEMS Microbiol. Lett. 49, 389-392.

Veumann, A. W., Cood, R. J., Hope, C. J. \& Seipal, M., 1974. An equation-of-state approach to determine surface tensions of low-energy solids from contact angles. - J. Coll. Inter. Sci. 49. 291-302.

Veumann, A. W., Ahsolom, D. R., Francis, D. W. \& van Oss, C. J., 1980. Conversion tables of contact angles to surface tensions. - Sep. Purif. Methods 9, 69-163.

Parker, N. D. \& Munn, C. B., 1984. Increased cell surface hydrophohicity associated with possession of an additional surtace protein by Aeromonas salmonicida. - FE:MS Microbiol. Lett 21 . $233-2.37$

Paul, J. H. \& Jeffrey, W. H. 1985. Evidence for separate adhesion mechanisms for hydrophilic and hydrophobic surfaces in Vibrio proteolytica. - Appl. environ. Microbiol. 50, 431-437.

Pelt van, W. J., Weerkamp, A. H., Uyen, M. H. W. J. C., Bussher, H. J., de Jong, H. P. \& Arends, J.. 1985. Adhesion of Streptococcus sanguis CH3 to polymers with different surface free energies. - Appl. environ. Microbiol. 49, 1270-1275.

Pringle, J. H. \& Fletcher, M., 1983. Influence of substratum wettability on attachment of freshwater bacteria to solid surfaces. - Appl. environ. Microbiol. 45, 811-817.

Rabel, W. 1971. Einige Aspekte der Benetzungstheorie und ihre Anwendung auf die Untersuchung und Veränderung der Oberflächeneigenschaften von Polymeren. - Farbe und Lack. 77, 997-1005

Rittle, K. H., Helmstetter, C. E., Meyer, A. E. \& Baier, R. E., 1990. Escherichia coli retention on solid surfares as functions of substratum surface free energy and cell growth phase. - Biofouling 2. $121-130$.

Rittschof, D. \& Costlow, J. D., 1989. Bryozoan and barnacle settiement in relation to initial surface wettability: A comparison of laboratory and field studies. In: Topics in marine biology. Ed. by $E$. D. Ros. - Scientia mar. 53, 411-416.

Roberts, D., Rittschof, D., Holm, E. \& Schmidt, A. R., 1991. Factors influencing initial larval-settlement: teinporal, spatial and surface molecular components. - J. exp. mar. Biol. Ecol. 150 , 203-211.

Rutter, P. R, 1980. The physical chemistry of the adhesion of bacteria and other cells. In: Cell adhesion and mobility. Ed. by A. S. G. Curtis \& J. D. Pitts. Cambridge Univ. Press, London. 103-135.

Shed, C., Nunley, J. W. Williamson, J. C. \& Smith-Sommerville, H. E., 1991. Comparison of the adhesion properties of Deleya marina and the exopolysaccharide-defective mutant strain DMR. - Appl. environ. Microbiol. 57, 3107-3113. 
Sutherland, I. W., 1980. Polysaccharides in the adhesion of marine and freshwater bacteria. In: Microbial adhesion to surtaces. Ed. by R. C. W. Berkeley, J. M. Lynch, J Melling, R. P. Rutter \& B. Vincent. Horwood. Chichester, 330-338.

Udhayakumar, M. \& Karande, A. A., 1986. Adhesive strength of some biofouling organisms. Curr. Sci. 55. 656-658

Vogelbein, W. K. \& Thune, R. L., 1988. Ultrastructural features of three ectocommensal protozoa attached to the gills of the red swamp crawfish, Procambarus clarkii (Crustaced: Decapoda). - J. Protozool. 35, 341-348.

Webster, D. R., Cooksey, K. E. \& Rubin, R. W., 1985. An investigation of the involvement of cytoskeletal structures and secretion in gliding motility of the marine diatom. Amphora coffaeformis. - Cell Motility .5, 103-122.

Wigglesworth-Cooksey, B. \& Cooksey, K. E., 1992. Can diatoms sense surfaces?: State of our knowledge. - Biofouling 5, 227--238.

Witman, J. D. \& Suchanek, T. H., 1984. Mussels in flow: Drag and dislodgement by epizodns. Mar. Ecol. Prog. Ser. 16, 259-268.

Woodin. S. A., 1986. Settlement of infauna: Larval choice? - Bull, mar. Sci. 39. 401-407.

Woods, D. C. \& Fletcher, R. I., 1991. Studies on the strength of adhesion of some common marine fouling diatoms. - Biofouling 3, 287-303.

Wu. S., 1973. Polar and nonpolar interactions in adhesion. J. Adhesion 5. 39-5.5.

Yule, A. B. \& Crisp, D. J., 1983. Adhesion of cyprids of the larvae of the barnacle. Balanus balanoides, to clean and Athropodin treated surfaces. - J. mar. biol. Ass. L.K. 63, 261-271

Yule, A. B. \& Walker, $C_{2}, 1984$. The temporary adhesion of barnacle cyprids: Efferts of some diftering surface characteristıcs. - J. mar. biol. Ass. U.K. 64, 429-439.

Yule, A. B. \& Walker, C., 1985. Settienent of Baianus balanoides: The eitert of cyprid antennuiar secretion. - J. mar. biol. Ass. U.K. 65, 707-712.

Zisman. W. A., 1964. Relationships of equilibrium contact angle to liquid and solid constitution. Adv. Chem. 43. American Chemical Society. Washington DC., 1-51. 